\title{
PERTURBED SITES AND HOST-GUEST-HOST EXCITON CASCADE IN THE BIPHENYL ISOTOPIC MIXED CRYSTAL, PHOSPHORESCENCE
}

\author{
Peter S. FRIEDMAN, Paras N. PRASAD * and Raoul KOPELMAN \\ Department of Chemistry, The University of Michigan, Ann Arbor, Michigan 48104, USA
}

Received 18 August 1974

Revised manuscript received 14 November 1975

\begin{abstract}
An interesting energy cascade is observed in the phosphorescence spectra of $1 \%$ biphenyl-h $h_{10}$ in biphenyl- $d_{10}(2-15 \mathrm{~K})$; strongly perturbed host sites, with energy levels below that of the protonated guest, quench the guest sites at higher temperatures $(11-15 \mathrm{~K})$. The identification of the perturbed sites is based on vibrational characteristics (both intensity and frequency), obtained with the help of phosphorescence spectra of biphenyl- $h_{10}$ and biphenyl- $d_{10}$ in an argon matrix, indicating an isotope dependent vibronic structure. A partial vibrational analysis is presented, resulting in confimation of the first triplet state of biphenyl as orbitally ungerade. The dy namics of the triplet excitation are discussed, including several possible mechanisms explaining the non-Boltzmann nature of the low-temperature steady state.
\end{abstract}

\section{Introduction}

The biphenyl crystalline system has been investigated by several workers in recent years $[1-6]$. This system, involving a prototype non-rigid molecule, has been shown to exhibit very complex behavior in the crystalline phase, where an unusual continuous structural change has been observed [1] at low temperature $(\approx 20 \mathrm{~K})$ and some evidence has been given for disorder $[2-3]$. In this paper we present some investigations of the triplet state of the biphenyl crystal, where a rather unique energy transfer has been found. In dilute isotopic mixed crystals of biphenyl we have observed small concentrations of strongly perturbed biphenyl- $d_{10}$ host sites which have energy levels lower than that of the preponderant guest biphenyl- $h_{10}$ sites. Our interpretation is substantiated by the observed phosphorescence of biphenyl- $h_{10}$ and biphenyl- $d_{10}$ in an argon matrix. In both the argon and biphenyl environment the vibronic structure has a strong isotope dependence.

¥ Supported by NSF Grant GH-32578X and NIH Grant NS08116.

* Present address: Department of Chemistry, State University of New Yozk at Buffalo, Buffalo, New York 14214, USA.
The nature of the ordinary and perturbed sites is discussed in the context of excitation dynamics. In the triplet state of the isotopic mixed biphenyl crystals, the excitation seems to cascade to lower energy sites as the temperature is raised involving a nonBoltzmann behavior. The perturbed biphenyl- $d_{10}$ sites become the predominant energy traps for the triplet excitation as the temperature is raised from $2 \mathrm{~K}$ to $15 \mathrm{~K}$, while at $2 \mathrm{~K}$ only bipnenyl $-h_{10}$ is the emitting species.

The present work also confims the assignment of the symmetry species of the first triplet state, based on a vibrational analysis of the phosphorescence of biphenyl- $h_{10}$ and biphenyl- $d_{10}$.

\section{Experimental}

Biphenyl- $h_{10}$ was obtained from James Hinton (Virginia) and was of stated purity $99.99 \%$ (zone refined). In the latter part of the work we also used biphenyl obtained from Aldrich with $99.9 \%$ quoted purity, which we further purified by 80 passes of zone refining. Biphenyl- $d_{10}$ was obtained from Stohler Isotope Chemicals, Inc., and had $99.5 \%$ deuterium atoms. The isotopic mixed crystals were 
prepared by first placing the mixture in a hel: $1 \mathrm{~m}$ atmosphere, evacuating to $10^{-6}$ torr, melting, freezing and re-evacuating; the crystal growing tube was then seated off from the vacuum line and the sample was remelted and vigorously shaken. The isotopic mixed crystals were then grown slowily in a Bridgman furnace over a period of a few days; however, as we did not obtair. optically perfest single cystals, no attempt was made to orient them. The crystal sur. faces were polished smooth using an ethanol moistened chamois cloth.

The $2 \mathrm{~K}$ phosphorescence from $1 \%$ isotopic mixed_crystals was obtained photographically on a 1.0-meter 25-100 Czerny-Turner Jarrel-Ash spectrometer with a 1600 watt Xe lamp as the excitation source. A Kasha filter consisting of a 2:1 ratio solution mixture of $225 \mathrm{~g} / \mathrm{N} \mathrm{NiSO}_{4} \cdot 6 \mathrm{H}_{2} \mathrm{O}$ and $100 \mathrm{~g} / \mathrm{e}$ $\mathrm{CoSO}_{4} \cdot 7 \mathrm{H}_{2} \mathrm{O}$ in a $5 \mathrm{~cm}$ cell along with a Schott UG-11 filter were used to select the excitation. Second order nuorescence overlap was prevented by use of a Corning 0-52 filter. The spectrum was recorded on Kodak Type IIa-D film in the first order using a $50 \mu$ slit. The resolution was approximately $4 \mathrm{~cm}^{-1}$.

The phosphozescence of biphenyl, both $h_{10}$ and $d_{10}$, in an argon matrix was performed on a 1.5 meter Bausch and Lomb monochromator, at $5 \mathrm{~cm}^{-1}$ re. solution with a 1600 watt xenon lamp as the excitation source. A neat argon matrix was first deposited for approximately twenty minutes on the front surface of the quartz sample cell, which contained liquid helium on the inside. The temperature of the matrix was approximately $5 \mathrm{~K}$. The biphenyl sample was then heated in an oil bath to about $45-50^{\circ} \mathrm{C}$ and allowed to mix with the argon gas; the resulting mixture was then deposited on top of the neat argon matrix and allowed to build up to the desired sample thickness. The thickness of the biphenyl- $h_{10}$ matrix was about 1-2 min, while the matrix thick: ness for biphenyl- $d_{10}$ appeared to be about $0.5 \mathrm{~mm}$. The thinner matrix seemed to give less scattering; we note, however, that the Kasha filter (mentioned above) was twice as concentrated for the perdeuterated guest, which may have helped to cut down on the scattering. The incident illumination was directed straight through the sample (i.e., not at a right angle). Spectra were recorded on Kodak 103a-D film in firstorder using a $60 \mu$ slit. We note that phosphorescence was also attempted for biphenyl- $h_{10}$ in $p$-dibromobenzene; however, phosphorescence from the host obscured any possible emission from the guest.

A temperature dependence study of the phosphorescence from the isotopic mixed biphenyl was conducted in a 10DT helium injection type Janis cryostat equipped with a temperature control unit. The temperature was measured by using Ge resistors. Thesc spectra were obtained on a 1 meter JarrelAsh with photoelectric recording by a dc detection system. The excitation source was that 100 watt medium pressure mercury lamp.

\section{Results}

Fig. 1 shows a densitometer tracing of the phosphorescence from a $1 \%$ isotopic mixed biphenyl crystal at $2 \mathrm{~K}$. It can be seen that the spectrum consists of a doublet having a spacing of approximately $70 \mathrm{~cm}^{-1}$ at each band. Both the spacing and the intensity of these doublets do not change in going from one vibronic band to another. It is also evident that the origin is the strongest band in the spectrum.

Figs. 2 and 3 show, respectively, the phosphorescence of biphenyl- $h_{10}$ and biphenyl- $d_{10}$ in the argon matrix [7]. The bands are, in general, broader than in the isotopic mixed crystal phosphorescence. One can also notice that the protonated and deuterated biphenyl phosphorescence spectra do not have the same vibronic pattern (see vibronic analysis - tables 1 and 2). Fig. 4 compares the phosphorescence of the biphenyl isotopic mixed crystal at several temperatures. As the temperature is raised from $2 \mathrm{~K}$ to $11 \mathrm{~K}$, we see that the original doublet structures become considerably weaker. The higher energy component is not even observable at $11 \mathrm{~K}$; while some new multiplet structures have appeared on the lower energy side; also all of the major vibronic bands appear to be built on the new 0-0 multiplet. As the temperature is raised from $11 \mathrm{~K}$ to $15 \mathrm{~K}$ all these multiplet structures are replaced by a single (though broad) feature at every vibronic band. 


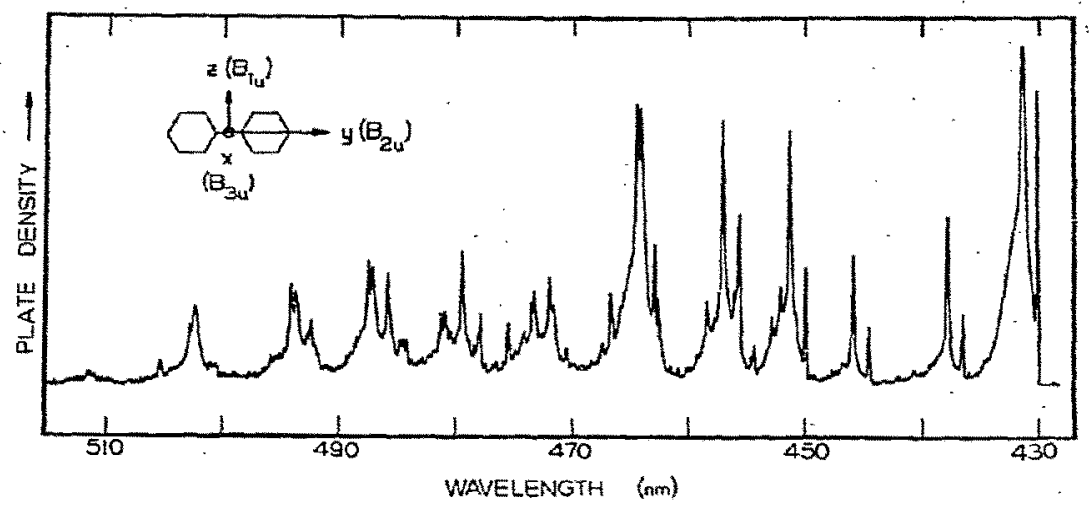

Fig. 1. Phosphorescence spectrum of $1 \%$ biphenyl- $h_{10}$ in biphenyl-d ${ }_{10}$ at $2 \mathrm{~K}$.

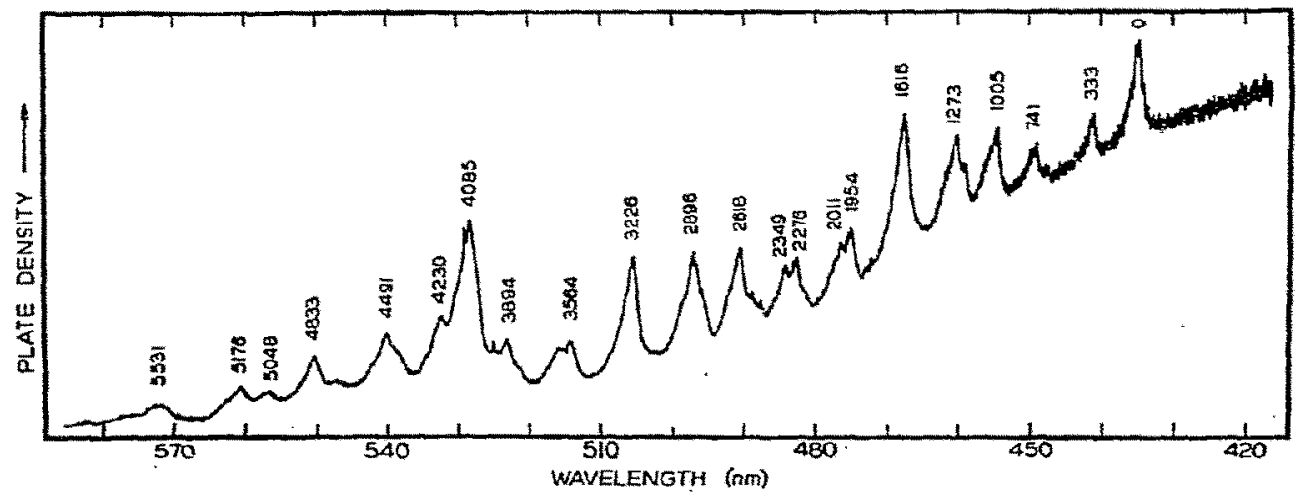

Fig. 2. Phosphorescence spectrum of biphenyl- $h_{10}$ in argon at $5 \mathrm{~K}$.

\section{Discussion}

\subsection{Biphenyl in argon}

As the phosphorescence spectrum of biphenyl in the argon matrix is the simplest one, we analyze this spectrum first so as to characterize the nature of the triplet states. The phosphorescence of biphenyl, both $h_{10}$ and $d_{10}$, in $n$-heptane at $77 \mathrm{~K}$ has recently been reported by $\mathrm{Lim}$ and $\mathrm{Li}[8]$. Our spectra of the two isotopes in the argon matrix (figs. 2 and 3 ) very much resemble the work by the above authors; however, our spectra appear somewhat sharper and better resolved. We note that in the biphenyl- $h_{10}$ spectrum a strong band is observed at about $5289 \AA$, which is not observed in the $n$-heptane spectrum and may therefore represent a possible impurity in the argon matrix. The vibrational analysis for the protonated and deuterated. guests appears in tables 1 and 2 . We note that the vibrational analysis indicates that all of the major bands involve only vibrations of the totally symmetric $\mathrm{a}_{\mathrm{g}}$ symmetry class; however, it appears that different $\mathrm{a}_{\mathrm{g}}$ 


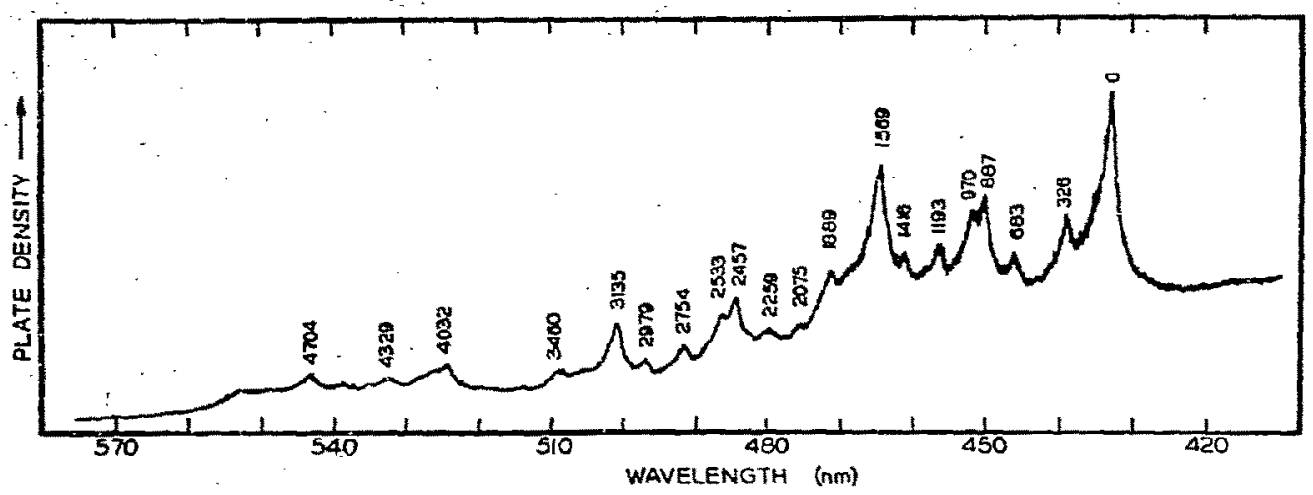

Fig. 3. Phosphorescence spectrum of biphenyl-d 10 in argon at $5 \mathrm{~K}$.

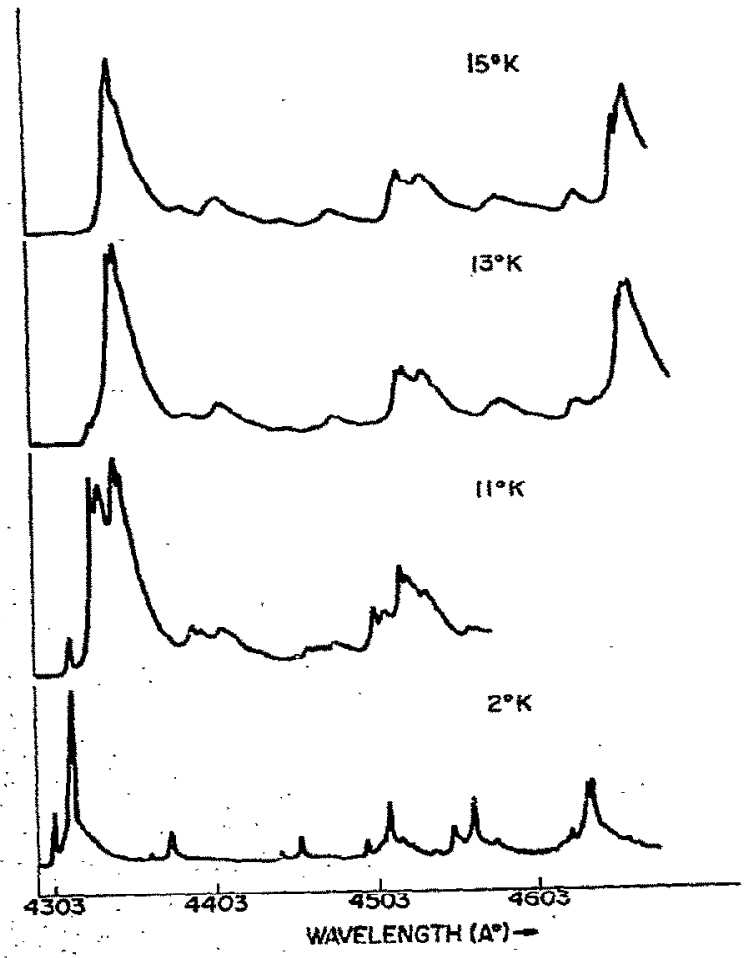

Fig. 4. Temperature dependence of the phosphorescence from a.crystal containing $1 \%$ biphenyl- $h_{10}$ in biphenyl- $d_{10}-$ fundamentals may be active in the biphenyl- $h_{10}$ and biphenyl- $d_{10}$ guest spectra. Such differences are not surprising to find. Several works $[9,10]$ have appeared recently which discuss the isotope dependence of vibronic structure.

The 0,0 appears to be very strong in the spectrum of both isotopes. This, coupled with the fact that the vibronic bands involve only $\mathrm{a}_{\mathrm{g}}$ vibrations, indicates that the triplet state has " $u$ " symmetry [11]. This is also borne out by the spectrum of the isotopic mixed crystal (see below) [11]. If we assume that the lowest biphenyl triplet state is derived from the lowest benzene triplet state, which is ${ }^{3} B_{1 u}$, then the orbital symmetry of the emitting triplet state of biphenyl should be ${ }^{3} B_{2 u}$ (see fig. 1) $[2,6]$.

\subsection{Biphenyl in biphenyl-d 10 at $2 \mathrm{~K}$}

Next we would like to discuss the low temperature ( $2 \mathrm{~K}$ ) isotopic mixed crystal phosphorescence. As the doublet spacing is approximately constant $\left(70 \mathrm{~cm}^{-1}\right)$ throughout the spectrum, we conclude that these structures do not correspond to impurity spectra. In other words, they belong to the same chemical and isotopic species. The possibility that they could be due to two different close lying triplet states is ruled out by the vibronic patterns of the two components, which are the same. This is not expected to be the case for two triplet excited states having different orbital symmetry. Further and conclusive evidence is 
Table 1

Vibrational analysis for phosphorescence of biphenyl- $h_{10}$ in argon ( $5 \mathrm{~K}$ )

\begin{tabular}{llrl}
\hline$\lambda(\AA)$ & $\left.\bar{v}\left(\mathrm{~cm}^{-1}\right)^{a}\right)$ & \multicolumn{1}{c}{$\Delta \bar{v}$} & Tentative assignment $\mathrm{b})$ \\
\hline 4349 & 22987 & 0 & 0,0 \\
4413 & 22654 & 333 & $334 \mathrm{a}_{\mathrm{g}}\left(v_{11}\right)$ \\
4494 & 22246 & 741 & $743 \mathrm{a}\left(v_{10}\right)$ \\
4548 & 21982 & 1005 & $1001 \mathrm{ag}_{\mathrm{g}}\left(v_{9}\right)$ \\
4604 & 21714 & 1273 & $1275 \mathrm{ag}_{\mathrm{g}}\left(v_{6}\right)$ \\
4678 & 21371 & 1616 & $1617 \mathrm{a}_{\mathrm{g}}\left(v_{4}\right)$ \\
4753 & 21033 & 1954 & $1951=1617+334$ \\
4766 & 20976 & 2011 & $2002=2 \times 1001$ \\
& & & $2018=1275+743$ \\
4827 & 20711 & 2276 & $2276=1275+1001$ \\
4844 & 20638 & 2349 & $2360=1617+743$ \\
4908 & 20369 & 2618 & $2618=1617+1001$ \\
4976 & 20091 & 2896 & $2892=1617+1275$ \\
5059 & 19761 & 3226 & $3234=2 \times 1617$ \\
5147 & 19423 & 3564 & $3568=2 \times 1617+334$ \\
5236 & 19093 & 3894 & $3893=1617+1275+1001$ \\
5289 & 18902 & 4085 & \\
5330 & 18757 & 4230 & $4235=2 \times 1617+1001$ \\
5405 & 18496 & 4491 & $4507=2 \times 1617+1273$ \\
5507 & 18154 & 4833 & $4851=3 \times 1617$ \\
5573 & 17939 & 5048 & \\
5613 & 17811 & 5176 & $5185=3 \times 1617+334$ \\
5727 & 17456 & 5531 & \\
\hline
\end{tabular}

a) Experimental accuracy is $\pm 15 \mathrm{~cm}^{-1}$.

b) Fundamental frequencies taken from B. Pasquier, Mol. Cryst. Liquid Cryst. 11 (1970) 35.

provided by our temperature study, given in detail below (and also by a recent heat pulse experiment [12] on the same samples, where an energy transfer from a high energy level to a low energy level with an increase in temperature is only consistent with the interpretation of these structures as being due to two sites). A vibrational analysis [2], based on the more intense components of the doublet, is presented in table 3. (We note that this analysis is in agreement with that just published by Bree et al. [23].) We see that the vibrational analysis correlates well with that of the phosphorescence of biphenyl- $h_{10}$ in the argon matrix (table 1) up to the totally symmetric $a_{\mathrm{g}}$ vibration, $v_{4}$; beyond this point the analysis becomes more complex. Additional OMDR work by Francis and Prasad [12] is in agreement with our interpretations.

Summarizing the above discussion we can say that
Table 2

Vibrational analysis for phosphorescence of biphenyl- $d_{10}$ in argon (5 K)

\begin{tabular}{|c|c|c|c|}
\hline$\lambda(\AA)$ & $\left.\bar{v}\left(\operatorname{cm}^{-1}\right)^{a}\right)$ & $\Delta \bar{v}$ & Tentative assignment b) \\
\hline 4332 & 23078 & 0 & 0,0 \\
\hline 4394 & 22752 & 326 & $\left.320^{c}\right) a_{g}\left(v_{11}\right)$ \\
\hline 4464 & 22395 & 683 & $688 \mathrm{a}_{\mathrm{g}}\left(\mathrm{v}_{10}^{\mathrm{g}}\right)$ \\
\hline 4505 & 22191 & 887 & $883 c)^{a_{g}\left(v_{8}\right)}$ \\
\hline 4522 & 22108 & 970 & $967 \mathrm{c}) \mathrm{a}_{\mathrm{g}}^{\mathrm{s}}\left(\mathrm{v}_{7}\right)$ \\
\hline 4558 & 21885 & 1193 & $1188 \mathrm{a}_{\mathrm{g}}\left(\mathrm{v}_{6}\right)$ \\
\hline 4615 & 21662 & 1416 & $1412 \mathrm{a}_{\mathrm{g}}^{\mathrm{B}}\left(v_{5}\right)$ \\
\hline 4648 & 21509 & 1569 & $1571 \mathrm{a}_{4 \mathrm{~g}}^{6}\left(v_{4}\right)$ \\
\hline 4718 & 21189 & 1889 & $1891=1571+320$ \\
\hline 4760 & 21003 & 2075 & $2071=1188 \div 883$ \\
\hline 4802 & 20819 & 2259 & $2254=1571+683$ \\
\hline 4848 & 20621 & 2457 & $2454=1571+883$ \\
\hline 4866 & 20545 & 2533 & $2538=1571+967$ \\
\hline 4919 & 20324 & 2754 & $2754=1571+1183$ \\
\hline 4974 & 20099 & 2979 & $2983=1571+1412$ \\
\hline 5013 & 19943 & 3135 & $3142=2 \times 1571$ \\
\hline 5096 & 19618 & 3460 & $3462=2 \times 1571+320$ \\
\hline 5249 & 19046 & 4052 & $4025=2 \times 1571+883$ \\
\hline 5332 & 18749 & 4329 & $4330=2 \times 1571+1188$ \\
\hline 5441 & 18374 & 4704 & $4713=3 \times 1571$ \\
\hline
\end{tabular}

a) Experimental accuracy is $\pm 10 \mathrm{~cm}^{-1}$.

b) Fundamental frequencies unless otherwise noted are from G. Zerbi and S. Sandroni, Spectrochim. Acta 24A (1968) 483.

c) Fundamental frequencies based on our data at $100 \mathrm{~K}$ (ref. [2]).

the two structures in the $2 \mathrm{~K}$ spectrum belong to two energetically inequivelant sites of biphenyl- $h_{10}$ in the biphenyl- $d_{10}$ lattice. From the room temperature crystal structure [13] of biphenyl, one does not expect such energetically inequivalent sites in isotopic mixed biphenyl, and thus it is possible that they represent either: (a) the "genuine" substitutional site and the site of an X-trap (i.e. a biphenyl- $h_{10}$ sitting next to a chemical impurity), or (b) both being due to $x$-traps. It has been shown [14] that when purified biphenyl is doped with a chemical impurity like carbazole or dibenzothiophene, biphenyl $\mathrm{x}$-traps are created and the phosphorescence is characteristic of such $x$-traps. The chemical impurities found in zone refined commerical biphenyl are mainly carbazole and anthracene [14] (and perhaps [14] phenanthrene). If carbazole is the chemical impurity in biphenyl, then the fluorescence should come from carbazole $[6,14]$. Our fluorescence spectra from neat 
Table 3

Vibrational analysis for phosphorescence of biphenyl- $h_{10}$ in biphenyl- $d_{10}$ at $2 \mathrm{~K}$

\begin{tabular}{|c|c|c|c|}
\hline$\lambda(A)$ & $\left.\bar{v}\left(\mathrm{~cm}^{-1}\right)^{\mathrm{a}}\right)$ & $\Delta \bar{v}$ & Tentative assignment $b$ ) \\
\hline 4303 & 23233 & -70 & defect (assumed) \\
\hline 4316 & 23163 & 0 & 0,0 (assumed) \\
\hline 4381 & 22819 & 344 & $334 \mathrm{a}_{\mathrm{g}}\left(v_{11}\right)$ \\
\hline 4459 & 22420 & 743 & $743 \mathrm{a}_{\mathrm{g}}\left(v_{10}\right)$ \\
\hline .4513 & 22152 & 1011 & $1001 \mathrm{a}_{g}\left(v_{9}\right)$ \\
\hline 4569 & 21880 & 1283 & $1275 \mathrm{a}_{\mathrm{g}}\left(u_{6}\right)$ \\
\hline 4640 & 21546 & 1617 & $1617 \mathrm{ag}_{\mathrm{g}}\left(v_{4}\right)$ \\
\hline
\end{tabular}

a) Experimental accuracy is $\pm 10 \mathrm{~cm}^{-1}$.

b) Fundamental assignments taken from B. Pasquier, Mol. Cryst. Liquid Cryst. 11 (1970) 35.

biphenyl- $d_{10}$ and from the isotopic mixed crystal $\left(h_{10}\right.$ in $\left.d_{10}\right)$ are nearly identical to those reported by Hochstrasser et al. [4], who have purified their biphenyl with extreme care (potassium fusion etc.). Their phosphorescene spectra (Hochstrasser, private communication), however, exclude the lower energy ("defect") set of our vibronic bands, thus being consistent with either of our above possibilities (a) or (b). The very long lifetime of the triplet state obviously makes the appearance of $\mathrm{x}$-trap emission more likely than for the singlet state. This situation is further underscored below.

\subsection{Temperature study and assignment of sites}

We now analyze our temperature dependence study. The $15 \mathrm{~K}$ spectrum appears to have only one major component and will be analyzed first because of this simplifying feature. This spectrum is built on a new origin, which does not exist in the $2 \mathrm{~K}$ spectrum. The two origins of the $2 \mathrm{~K}$ spectrum have totally lost their intensity and the vibronic structures built on them have disappeared. The vibrational analysis (table 4) shows that (except for the lowest mode at $330 \mathrm{~cm}^{-1}$, see below) every band clearly corresponds to a vibrational frequency of biphenyl- $d_{10}$ (and not to biphenyl $h_{10}$, which is the emitter of the $2 \mathrm{~K}$ phosphorescence). This assignment is further supported by the fact that the vibronic structurc appearing in the spectrum is quite similar, even intensity-wise, to that of the biphenyl- $d_{10}$-in-argon-matrix phosphorescence. Here it should be remembered that the vibronic. structure, in the argon inatrix phosphorescene dis- cussed above, shows a strong isotope dependence. The lowest mode $\left(\approx 330 \mathrm{~cm}^{-1}\right)$ appears to vary for the different site origins. This mode is a non-benzenoid (ring-ring stretch, $a_{g}$ ) soft mode and such modes are expected to be quite sensitive to crystal perturbations. In fact, a Raman study $[2,15]$ reveals that the frequency of this mode shows perhaps the largest temperature dependence of all biphenyl Raman modes.

In the $11 \mathrm{~K}$ spectrum the "new" origin (compared to $2 \mathrm{~K}$ ) consists of a multiplet structure, the prominent features again corresponding to biphenyl- $d_{10}$. The $13 \mathrm{~K}$ spectrum is in-between the $11 \mathrm{~K}$ and $15 \mathrm{~K}$ spectra and shows that intensity has been transferred from the higher energy sites to the lower energy sites. From this it appears that there are a number of biphenyl- $d_{10}$ sites: first, the preponderant (most probable but "quenched") sites have a concentration close to $100 \%$, which we denote by $C_{D}$ ( and the corresponding biphenyl- $h_{10}$ sites a concentration close to $1 \%$, denoted by $C_{H}$ ); also, there are smali concentrations of other sites with much lower triplet exciton energies which we designate $C_{\mathrm{D}}^{*}$ for biphenyl- $d_{10}$ (and $C_{\mathrm{H}}^{*}$ for biphenyl- $h_{10}$ ). Thus the temperature dependence study of the isotopic mixed crystal phosphorescence is consistent with the following picture: $C_{\mathrm{D}}>C_{\mathrm{H}}>C_{\mathrm{D}}^{*}>C_{\mathrm{H}}^{*}$, while the corresponding site energies presumably are $E_{\mathrm{D}}>E_{\mathrm{H}}>E_{\mathrm{D}}^{*}>E_{\mathrm{H}}^{*}$ (each energy covering a finite range, but not necessarily a continuous one). Also, as biphenyl- $d_{10}$ is the host, i.e. it contains only small amounts $(1 \%)$ of biphenyl- $h_{10}, C_{\mathrm{H}}^{*}$ must obviously be very small. Thus, when the excitation starts cascading to lower traps as the temperature is raised, it effectively stops at the $C_{\mathrm{D}}^{*}$ sites because of the very small concentration $C_{\mathrm{H}}^{*}$. This explains why the transition corresponding to $E_{\mathrm{H}}^{*}$ was not observed.

\subsection{Cause of the perturbed sites}

We have here a very unusual case insofar as a perdeutero site energy has been found to be lower than a perproto site energy; so far we are not aware of any published precedence. It cautions une not to always assign the lowest energy emission from an isotopic mixed crystal to the perproto species, not even in a "deep trap" case. We emphasize again that the preponderant sites in the crystal and in the argon matrix 
Table 4

Comparison of vibronic structures at $2 \mathrm{~K}$ and $15 \mathrm{~K}$

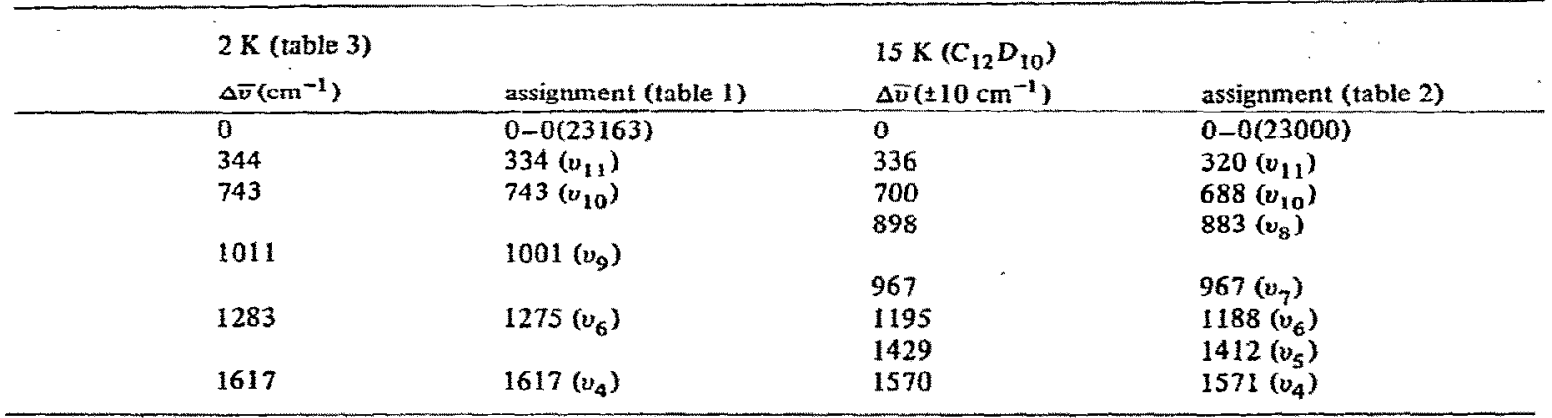

have the usual order $\left(E_{\mathrm{D}}>E_{\mathrm{H}}\right)$ and that the trapdepth $\left(91 \mathrm{~cm}^{-1}\right)$ is most probably large compared to the exciton bandwidth [16] . Again we emphasize that we are not able here to differentiate between perturbed sites that are $\mathrm{X}$-traps and other defect sites (possibly related to structural disorders or surface effects). Dynamically they have a similar role.

\subsection{The energy transfer}

We now come to a more detailed consideration of the nature of the temperature effects. Our previous data [1] indicate that the continuous phase transition starting at about $70 \mathrm{~K}$ may be mostly over (using Raman spectra as criterion) at $15 \mathrm{~K}$. While siructural changes originating in this phase transition are likely to be responsible for the perturbed sites giving rise to our observed spectra $(2-15 \mathrm{~K})$, we do not consider it as necessarily true that addi. tional subtle structural changes in the $2-15 \mathrm{~K}$ region are responsible for the major features of our observed phosphorescence temperature effect in this region. We therefore focus our attention on the dynamics of the triplet excitation. Obviously, our data cannot be explained by triplet excitons, in a quasi-Boltzmann equilibrium involving the various trap (and host) states, as long as we do not believe in a progressive creation with temperature of the low energy traps. The Boltzmann distribution model, which has recently been discussed by Fayer and Harris [17], would obviously result in increased emission from higher energy trap or host states with rising temperatures. The opposite is observed. We are left with the following alternative explanations for attaining a steady state that is closer to themal quasi-equilibritum at incresing.temperature $(15 \mathrm{~K})$ : (a) thermal detrapping of relatively shallow $\left(C_{H}\right)$ traps (trap-depth "within reach" by $k T$, within the triplet lifetime), followed by host exciton migration as investigated by Hutchison, Hirota and co-workers [18]. We note that the host exciton migration might also be influenced by temperature. (b) A temperature dependent exciton-phonon interaction, where the latter determines the trapping efficiency of the various traps, as suggested by us elsewhere [19]. This change in trapping efficiency might be responsible for the whole temperature effect or might assist the above (a) mechanism or the one below (c). Excitonphonon coupling might also influence host exciton migration (a). (c) A temperature dependent superexchange [20] interaction, changing the trap-to-trap migration [21] drastically, resulting in exciton percolation [22]. Any such effect will probably again be dominated by the temperature effect on the exciton-phonon coupling (b) and its interplay with the energy denominators involved in the superexchange interaction [20].

Due to the uncertainties in the trap-depths of the various traps (i.e. not knowing the exact location of the host triplet band), as well as the possible involvement of structural anomalies, we do not deem it fruitful to attempt a quantitative description of the thermal detrapping (a), of of any of the other factors, mentioned above $(b, c)$. However, the situation is probably analogous to that involving chemical impurities in the biphenyl host [18], and the one involving 
isotopic impurities in naphthalene [19]. The latter case is free of some of the complications found here, and stili appears to be quite similar; it is [19] dominated by mechanism (a), with possible secondary roles played by (b) and (c), as is the first case [18] . A quantitative determination of activation energies awaits a more detailed phosphorescence temperature study.

\section{Acknowledgement}

We would like to thank Professor A.H. Francis for his help and his permission to do the temperature dependence work in his laboratory. We also thank Professor T.M. Dunn and Dr. M. Spolitti for their aid with the argon matrix work. We gratefully acknowledge a preprint by Hochstrasser, Scott and Zewail, which was received after this manuscript was completed.

\section{References}

[1] P.S. Friedman, R. Kopelman and P.N. Prasad, Chem. Phys. Letters 24 (1974) 15.

[2] P.S. Friedman, Ph.D. Thesis, The University of Michigan, (1974).

(3) H.C Brenner, C.A. Hutchison Jr. and M.D. Kemple, J. Chem. Phys. 60 (1974) 2180.

(4) R.M. Hochstrasser, R.D. McAlpine and J.D. Whiteman, J. Chem. Phys. 58 (1973) 5078.

[5] R.M. Hochstrasser, Ho-Nan Sung and J.E. Wessel, J. Chem. Phys. 58 (1973) 4694.

I6I T.M. Misra and K. Mandal, J. Chem. Phys. 59 (1973) 2409.

[7] While in the isotopic mixed crystal at $2 \mathrm{~K}$ the phosphorescence and the fluorescence are of comparable intensity, the biphenyl fluorescence in the argon matrix was not observed. This leads us to suspect that the intersystem. crossing is considerably enhanced in the argon matrix.

[8] E.C. Lim and Y.H. Li, J. Chem: Phys. $\$ 2$ (1970) 6416.
[9] G. Orlandi and $W$. Siebrand, Chem. Phys. Letters 15 (1972) 465 .

[10] G. Orr and G.J. Small, Chem. Phys. Letters 21 (1973) 395.

[11] Biphenyl fuorescence, which is g+g (in $D_{2 h}$ ), shows several false vibronic (u) origins (ref. [6]). We do not believe, however, that the entire phosphorescence spectrum of this crystal is built upon a single false vibronic (u) origin, without any trace of the true origin.

[12] A.H. Francis and P.N. Prasad, unpublished work.

[13] G. Robertson, Nature 19, 593 (1961)

J. Trotter, Acta Cryst. 14 (1965) 1135;

A. Hargreaves and S.H. Rizvi, Acta Cryst. 15 (1962) 365 .

[14] A. Bree and R. Zwarich, Mol. Cryst. Liquid Cryst. 5 (1969) 369 .

[15] P.N. Brasad and R. Kopelman, Chem. Phys. Letters 21 (1973) 505;

A. Bree, C.Y. Pang and L. Rabeneck, Spectrochim. Acta 27A (1971) 1293 .

[16] If the ratio of the lowest singlet exciton bandwidths between benzene and biphenyl can be used as a guide, then the triplet bandwidth of biphenyl is of the order of $1 \mathrm{~cm}^{-1}$ or less.

[17] M.D. Fayer and C.B. Harris, Phys, Rev. 9B (1974) 748.

[18] N. Hirota and C.A. Hutchinson, J. Chem. Phys. 42 (1965) 2869;

N. Hirota, J. Chern.Phys. 43 (1965) 3354;

E.T. Harrigan and N. Hirota, J. Chem. Phys. 49 (1968) 2301.

See also: H.C. Brenner and C.A. Hutchison, J, Chem. Phys. 58 (1973) 1328; 59 (1973) 2172;

H.C. Brenner, J. Chem. Phys. $\$ 9$ (1973) 6362.

[19] F.W. Ochs, P.N. Prasad and R. Kopelman, unpublished; F.W. Ochs, Ph.D. Thesis, University of Michigan (1974).

[20] H.K. Hong and R. Kopelman, Phys. Rev. Letters 25 (1970) 1030; J. Chem. Phys. 55 (1971) 724.

[21] G.W. Robinson and R.P. Frosch, J. Chem. Phys. 38 (1963) 1187 ;

H. Sternlicht, G.C. Nieman and G.W. Robinson, I. Chem. Phys. 38 (1963) 1326.

[22] H.K. Hong and.R. Kopelman, J. Chem. Phys. 55 (1971) 5380;

K. Kopelman, E. Monberg, F.W. Uchs and P.N. Prasad, j. Chem. Phys. 62 (1975) 292; Phys. Rev. Letters 34 (1975) 1506 .

[23] A. Bree, M. Edelson and R. Zwarich, Chem. Phys. 8 (1975) 27 